\title{
A note on Q-algebras and quantization
}

\author{
Xiang Tang*
}

February 7, 2008

\begin{abstract}
In this note, we study Schwarz's conjecture on application of Q-algebras to strict quantization. We prove that in the case of a torus with a constant Poisson structure, Schwarz's formalism gives the same star product as Rieffel [10]. We construct twisted Fock modules as examples of quantization dg-modules in the case of a compact Kähler manifold. In particular, we relate this construction on $\mathbb{C P}^{1}$ to representations of a fuzzy sphere.
\end{abstract}

\section{Introduction}

The notion of a Q-algebra was introduced by Albert Schwarz [13] in the study of noncommutative supergeometry and gauge theory.

The idea of noncommutative geometry goes back to algebraic geometry and the famous Gelfand-Naimark theorem. All geometric properties of a closed smooth manifold $X$ are encoded in the algebra $C^{\infty}(X)$ of smooth functions on $X$, and a map from $X$ to another closed smooth manifold $Y$ defines an algebra homomorphism from $C^{\infty}(Y)$ to $C^{\infty}(X)$. This correspondence between geometric spaces and commutative algebras was extended to noncommutative geometry and algebras by Alain Connes and his followers 4]. Roughly speaking, in noncommutative geometry, every associative algebra is considered as an algebra of functions on a "noncommutative space." Noncommutative geometry studies the generalized notions of conventional geometry, and uses our geometric intuition to obtain a new and better understanding on some noncommutative (and even commutative) algebras, e.g. the algebras of functions on noncommutative tori.

One can say (not very precisely) that supergeometry is supercommutative noncommutative geometry. The algebra $C^{\infty}(X)$ of smooth functions on a supermanifold $X$ is a $\mathbb{Z}_{2}$-graded supercommutative algebra and a map between supermanifolds $X$ to $Y$ is an algebra homomorphism from $C^{\infty}(Y)$ to $C^{\infty}(X)$. In general, we can "define" a "noncommutative supermanifold" as a "space" having a $\mathbb{Z}_{2}$-graded associative (but not necessary

*Keywords: Q-algebra-Quantization-Poisson structure, Math. Classification: 53D55-46L65 
supercommutative) algebra of functions. Of course, this definition is as formal as the definition of noncommutative space in terms of an associative algebra of functions. It is safe to say that noncommutative supergeometry combines ideas of both noncommutative geometry and supergeometry.

A vector field on a supermanifold $X$ is a derivation on the supercommutative algebra $C^{\infty}(X)$. An odd vector field corresponds to an odd derivation, which changes the parity of an element in $C^{\infty}(X)$.

Definition 1.1 A supermanifold equipped with an odd vector field $Q$ obeying $Q^{2}=0$ is called a $Q-$ manifold.

A natural generalization of Definition 1.1 to noncommutative geometry leads to the following definition.

Definition 1.2 A Q-algebra $A$ is a $\mathbb{Z}_{2}$ (or $\mathbb{Z}$ ) graded associative algebra with a derivation $Q$ of degree 1 and an element $\omega \in A$ of degree 2 satisfying

$$
Q^{2} x=[\omega, x]
$$

for all $x \in A$.

We call $\omega$ the curvature of the Q-algebra $A$.

Schwarz [13, 14] studied the theory of modules over a Q-algebra and proved a general duality theorem in this framework which generalized the $S O(d, d ; \mathbb{Z})$ duality of gauge theories on noncommutative tori to Q-algebras. In particular, he discovered an interesting connection between $Q$-algebras and deformation quantization.

The problem of formal deformation quantization of a general Poisson manifold was solved by Kontsevich [9]. Kontsevich's construction implicitly used the topological BV sigma-model [1], which was formulated by Cattaneo and Felder [3]. However, in Kontsevich's star product, the parameter $\hbar$ is a formal one. The problem of whether one can make $\hbar$ into a real number and construct a continuous family of associative multiplications is much harder. Using Q-algebras, Schwarz [14] suggested a way that allows one to circumvent the above strict quantization problem. We explain this idea in the following paragraphs.

We recall Fedosov's method of formal deformation quantization of symplectic manifolds. One starts with a standard formal Weyl algebra $W$ corresponding to a symplectic vector space, and constructs a Weyl algebra bundle $\mathcal{W}$ over a symplectic manifold $X$ through the principal symplectic group bundle. A torsion free symplectic connection on $X$ lifts to a connection $\nabla$ on bundle $\mathcal{W}$, which determines a structure of a Q-algebra on the algebra of $\mathcal{W}$-valued differential forms $\Omega(X, \mathcal{W})$ with curvature ${ }^{1}$ equal to $R$. Fedosov's results on deformation quantization can be reformulated into the property that the Q-algebra $(\Omega(X, \mathcal{W}), \nabla, R)$ is equivalent to a new Q-algebra $(\mathcal{A}, D, \nu)$ such that the

\footnotetext{
${ }^{1}$ The curvature of a right module $M$ over a Q-algebra $(A, Q, \omega)$ with a connection $\nabla$ is defined to be $\nabla^{2}+\hat{\omega} \in \operatorname{Hom}(M)$, where $\hat{\omega}$ is the right multiplication of $\omega$.
} 
curvature $\nu$ is equal to $-\frac{i}{\hbar} \omega+\tilde{\omega}$, where $\omega$ is the symplectic form on $X$, and $\tilde{\omega}$ is a 2-cocycle in $\Omega^{2}(X, \mathbb{C}[[\hbar]])$. (Two Q-algebras are equivalent if they are isomorphic and their derivations are related by the formula $Q^{\prime}=Q+[\gamma$,$] , for \gamma \in A$ ). This equivalent Q-algebra can be viewed as a differential graded algebra $\left(\mathcal{A}^{\prime}, D\right)$ because its curvature $\nu$ is a center element. It is proved by Fedosov [6] that the differential graded algebra $\left(\mathcal{A}^{\prime}, D\right)$ is quasi-isomorphic to an associative algebra $A(X)$ that can be considered as a quantization of $X$. (Notice that $A(X)$ is isomorphic to $C^{\infty}(X)[[\hbar]]$ as a vector space, and the product on $A(X)$ is translated to a star product on $C^{\infty}(X)[[\hbar]]$.) Since $\left(\mathcal{A}^{\prime}, D\right)$ is quasi-isomorphic to $A(X)$, they share the same geometric properties, i.e. cohomology, characteristic classes, and K-theory, etc. In particular, the category of projective modules of $A(X)$ is equivalent to the category of projective dg-modules of $\left(\mathcal{A}^{\prime}, D\right)$ up to quasi-isomorphisms. (A dg-module over a differential graded algebra is a module with a flat connection.) It is easy to check that there is a one-to-one correspondence between projective dg-modules of the differential graded algebra $\left(\mathcal{A}^{\prime}, D\right)$ and projective modules of the Q-algebra $(\mathcal{A}, D, \nu)$ with curvature equal to the multiplication of $\nu$. By the equivalence between the Q-algebras $(\Omega(X, \mathcal{W}), \nabla, R)$ and $(\mathcal{A}, D, \nu)$, there is again a one-to-one correspondence between the projective modules with the fixed curvature $\hat{\nu}$. We conclude that projective modules of $A(X)$, the deformation quantization of $X$, are related to projective modules of $(\Omega(X, \mathcal{W}), \nabla, R)$ with curvature equal to $\hat{\nu}$. We emphasize that it is important to work with the Q-algebra $(\mathcal{A}, D, \nu)$ instead of just the differential algebra $\left(\mathcal{A}^{\prime}, D\right)$.

Schwarz's crucial observation [14] is that the parameter $\hbar$ in $\Omega(X, \mathcal{W})$ can be made into a real number, so that $\Omega(X, \mathcal{W})$ becomes a complete topological algebra. This is achieved by an appropriate modification of the notion of Weyl algebra, which becomes a complete nuclear local convex algebra depending smoothly on the real parameter $\hbar$. Then one can consider a "new" Weyl algebra bundle $\mathcal{W}$, and a "topological" $Q$-algebra $(\Omega(X, \mathcal{W}), \nabla, R)$ as the space of sections of $\mathcal{W}$. It is possible that one can find an equivalent Q-algebra $(\mathcal{A}, D, \nu)$ such that the curvature $\Omega$ is in the center of $\mathcal{A}$, and the associated differential graded algebra $\left(\mathcal{A}^{\prime}, D\right)$ is quasi-isomorphic to an associative topological algebra $A(X)$, which is isomorphic to $C^{\infty}(X)$ as a vector space. Then the algebra $A(X)$ can be considered as a strict deformation quantization of $X$. We should remark that it is very hard (maybe impossible) to construct a topological Q-algebra $(\mathcal{A}, D, \nu)$ satisfying the above requirements. However, Schwarz [14] suggested that instead of searching for such an equivalent differential graded algebra, one should study the Q-algebra $(\Omega(X, \mathcal{W}), \nabla, R)$ directly. He conjectured that the Q-algebra $(\Omega(X, \mathcal{W}), \nabla, R)$ can be considered as a replacement of a convergent deformation quantization. In particular, if a strict deformation quantization of $X$ exists, the category of projective modules of the Q-algebra $(\Omega(X, \mathcal{W}), \nabla, R)$ with curvature equal to $\nu$ should be related to the category of projective modules of the strict deformation quantization of $X$. Schwarz conjectured that if the strict deformation quantization does not exist, the above category of modules should be regarded as a replacement of the category of projective modules of the strict deformation quantization. In any case, by studying this Q-algebra and its modules, we 
may obtain the information of the strict deformation quantization of $X$. Furthermore, this study may also lead to some "no go" theorems in strict deformation quantization, which should be very interesting.

The results in this paper have two important improvements with respect to Schwarz's original work 14. Firstly, to realize the above proposal, we need to know how to modify the formal Weyl algebra into a "nice" topological algebra. This question has many different possible answers. Schwarz [14] suggested one candidate for the answer. We find that the proposed algebra is hard to work with in the case of noncommutative tori. Gracia-Bondia and Várilly [7] studied the largest possible *-subalgebra of tempered distributions on $\mathbb{R}^{2 n}$ where the "twisted product" (convolution product) is defined. This *-subalgebra is invariant under Fourier transform and is very "big", and contains rapidly decreasing smooth functions, distributions of compact support and all polynomials, etc. Later, Dubois-Violette, Kriegl, Maeda and Michor [5] considered a smaller subalgebra $\mathcal{O}_{M}^{\prime}\left(\mathbb{R}^{2 n}\right)$ of speedily decreasing distributions on $\mathbb{R}^{2 n}$, whose definition will be recalled in Section 2.1. They suggested that $\left(\mathcal{O}_{M}^{\prime}\left(\mathbb{R}^{n}\right), \hat{*}_{\hbar}\right)$ be viewed as the covering space of a noncommutative torus $\mathcal{T}_{\theta}$, where $\theta$ is a constant Poisson structure on an $n$-dimensional torus. In this paper, we will follow this suggestion and use the subalgebra $\left(\mathcal{O}_{C}, *_{\hbar}\right)$, the Fourier transform of $\left(\mathcal{O}_{M}^{\prime}\left(\mathbb{R}^{n}\right), \hat{*}_{\hbar}\right)$, as the fiber of the new Weyl algebra. The good property of the algebra $\mathcal{O}_{C}$ is that if $f \in \mathcal{O}_{C}$ and $d f=0$, then there is $g \in \mathcal{O}_{C}$ with $d g=f$. Therefore the de Rham differential is exact on $\mathcal{O}_{C}$.

Secondly, we recall that in [14] a dg-module over a Q-algebra $(A, Q, \omega)$ is defined to be an $A$-module $M$ with a connection $\nabla_{M}$ such that the curvature $\nabla_{M}^{2}+\hat{\omega}$ equals 0 as an endomorphism on $M$, where $\hat{\omega}$ is the multiplication of $\omega$ on $M$. This is a natural generalization of the notion of a dg-module over a differential graded algebra. However, this definition is not very useful for our purpose. As described above, in Fedosov's formal deformation quantization of a symplectic manifold, projective modules of the associative algebra $A(X)$ are related to projective modules of the Q-algebra $(\Omega, \nabla, R)$ with curvature equal to $\nu$. This experience suggests that we should allow a dg-module over a Q-algebra to have a central curvature, not just 0 . Therefore, we introduce the following modified definition of a dg-module over a $Q$-algebra.

Definition 1.3 A dg-module over a Q-algebra $(A, Q, \omega)$ is a right $A$-module $M$ with a connection $\nabla_{M}$ such that the curvature $\nabla_{M}^{2}+\hat{\omega}$ as an element in $\operatorname{End}(M)$ is the multiplication by a center element in $A$.

In the case of a symplectic manifold $(X, \omega)$, we call a right $(\Omega(X, \mathcal{W}), \nabla, R)$-module a quantization dg-module if its curvature is equal to the multiplication by $-\frac{i}{\hbar} \omega$.

The goal of this paper is to study Schwarz's conjecture 14 on some examples. We prove that his conjecture is correct in these cases. The paper is arranged as follows. In Section 2, we study the case of a torus with a constant Poisson structure, and construct a Q-algebra $\left(\Omega\left(\mathbb{T}^{n}, \mathcal{W}\right), Q\right)$ with curvature equal to $-\frac{i}{\hbar} \omega$. We show that, viewed as a differential algebra, $\left(\Omega\left(\mathbb{T}^{n}, \mathcal{W}\right), Q\right)$ is quasi-isomorphic to the algebra of the corresponding quantum torus. By this quasi-isomorphism, we know that the algebra 
$\left(\Omega\left(\mathbb{T}^{n}, \mathcal{W}\right), Q,-\frac{i}{\hbar} \omega\right)$ contains as much information as a quantum torus. In Section 3 , we will construct a Fock module over $\Omega(X, \mathcal{W})$ on a compact Kähler manifold, which leads to examples of quantization dg-modules over $(\Omega(X, \mathcal{W}), \nabla, R)$. We show that in the case of a standard 2-sphere, our construction is connected to the well-known fuzzy sphere. We end this note with a conjecture on quantization dg-modules.

Acknowledgement: First of all, I would like to thank Albert Schwarz, who should be considered as a "virtual co-author", for many interesting questions and helpful suggestions. I also want to thank Alan Weinstein for helpful conversations.

\section{Quantum Tori}

In this section, we exhibit a construction for quantizing a constant Poisson torus following the idea described in the Introduction. We obtain the quantization in three steps:

1. quantization of $\mathbb{R}^{n}$;

2. Q-algebra;

3. quantization.

\subsection{Quantization of $\mathbb{R}^{n}$}

In this part, following the ideas of Dubois-Violette, Kriegl, Maeda and Michor [5], we introduce an algebra that plays the role of the Weyl algebra in the formal deformation quantization.

We recall here some well-known results in the distribution theory.

Let $\mathcal{S}\left(\mathbb{R}^{n}\right)$ be the space of rapidly decreasing smooth functions $f$ for which $x \mapsto$ $\left(1+|x|^{2}\right)^{k} \partial^{\alpha} f(x)$ is bounded for all $k \in \mathbb{N}$ with all multi-indices $\alpha \in \mathbb{N}_{0}^{n}$. We endow it with the locally convex topology described by these conditions, so that it is a nuclear Fréchet space. Let $\mathcal{S}^{\prime}\left(\mathbb{R}^{n}\right)$ be the dual space of $\mathcal{S}\left(\mathbb{R}^{n}\right)$ consisting of tempered distributions.

Let $\mathcal{O}_{C}\left(\mathbb{R}^{n}\right)$ be the space of all smooth functions $f$ on $\mathbb{R}^{n}$ for which there exists $k \in \mathbb{Z}$ such that $x \mapsto\left(1+|x|^{2}\right)^{k} \partial^{\alpha} f(x)$ is bounded for each multi-index $\alpha \in \mathbb{N}_{0}^{n}$. We endow this space with the locally convex topology. $\mathcal{O}_{C}\left(\mathbb{R}^{n}\right)$ is a complete nuclear (LP-) space. Let $\mathcal{O}_{C}^{\prime}\left(\mathbb{R}^{n}\right)$ be its dual space consisting of rapidly decreasing distributions.

Let $\mathcal{O}_{M}\left(\mathbb{R}^{n}\right)$ consists of all smooth functions on $\mathbb{R}^{n}$ such that for each multi-index $\alpha \in \mathbb{N}_{0}^{n}$, there exists $k \in \mathbb{Z}$ such that $x \mapsto\left(1+|x|^{2}\right)^{k} \partial^{k} f(x)$ is bounded. We endow it with the locally convex topology described by this condition. $\mathcal{O}_{M}$ is usually called the space of tempered smooth functions, whose dual space $\mathcal{O}_{M}^{\prime}\left(\mathbb{R}^{n}\right)$ is called the space of speedily decreasing distributions. 
We have the following relations among the above introduced spaces:

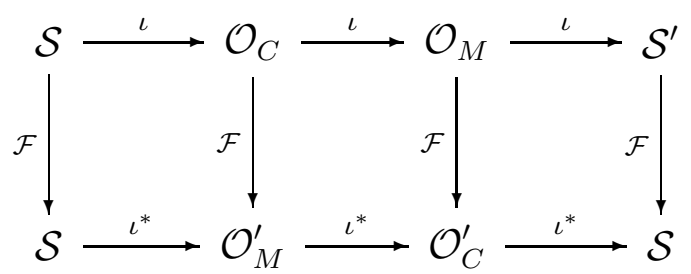

where in the above, $\iota$ is the embedding map, and $\mathcal{F}$ is the Fourier transform.

In Section 3, 5], the following theorem is proved.

Theorem 2.1 The following convolution

$$
\left(a \hat{*}_{\hbar} b\right)(x)=\int_{\mathbb{R}^{2}} a(x-y) b(y) e^{-\frac{i \hbar}{2} \omega(x, y)} \mathrm{d} y
$$

where $\omega$ is the standard symplectic two form on $\mathbb{R}^{2}$, defines a star product $\hat{*}_{\hbar}$. This product is an associative bounded multiplication on the space $\mathcal{O}_{M}^{\prime}\left(\mathbb{R}^{2}\right)$ of speedily decreasing distributions. It is smooth in the variable $\hbar \in \mathbb{R}$.

The following formula

$$
\hat{a} \stackrel{\text { def }}{=} \int_{\mathbb{R}^{2}} a(t, s) e^{\frac{i \hbar}{2} t s} e^{i t Q} e^{i s P} \mathrm{~d} s \mathrm{~d} t
$$

defines a bounded linear mapping $\mathcal{O}_{M}^{\prime}\left(\mathbb{R}^{2}\right) \rightarrow L(\mathcal{S}(\mathbb{R}), \mathcal{S}(\mathbb{R}))$, where $e^{\text {isP }} f(u) \stackrel{\text { def }}{=} f(u+s \hbar)$ and $e^{i t Q} f(u) \stackrel{\text { def }}{=} e^{i t u} f(u)$. This representation is injective if $\hbar \neq 0$, and is an algebra homomorphism from the product (1) to the operator composition. The analog on $\mathbb{R}^{2 n}$ also holds. Then

We calculate the pullback of $\hat{*}_{\hbar}$ to $\mathcal{O}_{C}$ through Fourier transformation. Let $f, g \in \mathcal{O}_{C}$.

$$
\begin{aligned}
& \mathcal{F}^{-1}\left(\mathcal{F}(f) \hat{\mathcal{*}}_{\hbar} \mathcal{F}(g)\right)(x) \\
= & \frac{1}{(2 \pi)^{2 n}} \int_{\mathbb{R}^{2 n}} e^{i x \cdot y} \mathcal{F}(f) \hat{\varkappa}_{\hbar} \mathcal{F}(g)(y) \mathrm{d} y \\
= & \frac{1}{(2 \pi)^{2 n}} \int_{\mathbb{R}^{2 n}} e^{i x \cdot y} \int_{\mathbb{R}^{2 n}} \mathcal{F}(f)(y-u) \mathcal{F}(g)(u) e^{-\frac{i \hbar}{2} \omega(y, u)} \mathrm{d} u \mathrm{~d} y \\
= & \frac{1}{(2 \pi)^{2 n}} \int_{\mathbb{R}^{2 n}} \int_{\mathbb{R}^{2 n}} e^{i x \cdot y-\frac{i \hbar}{2} \omega(y, u)} \mathrm{d} y \mathrm{~d} u \int_{\mathbb{R}^{2 n}} e^{-i(y-u) \cdot w} f(w) \mathrm{d} w \int_{\mathbb{R}^{2 n}} e^{-i u \cdot z} g(z) \mathrm{d} z \\
= & \frac{1}{(2 \pi)^{2 n}} \int_{\mathbb{R}^{2 n}} \int_{\mathbb{R}^{2 n}} \int_{\mathbb{R}^{2 n}} \int_{\mathbb{R}^{2 n}} e^{i x \cdot y-\frac{i \hbar}{2} \omega(y, u)-i(y-u) \cdot w-i u \cdot z} f(w) g(z) \mathrm{d} y \mathrm{~d} u \mathrm{~d} w \mathrm{~d} z \\
= & \int_{\mathbb{R}^{2 n}} \int_{\mathbb{R}^{2 n}} f(w) g(z) e^{\frac{-2 i}{\hbar} \omega(x-z, x-w)} \mathrm{d} z \mathrm{~d} w .
\end{aligned}
$$


Therefore, we define on $\mathcal{O}_{C}\left(\mathbb{R}^{2 n}\right)$ a product $*_{\hbar}$ by

$$
f *_{\hbar} g(x)=\frac{1}{(\pi \hbar)^{2 n}} \int_{\mathbb{R}^{2 n}} \int_{\mathbb{R}^{2 n}} f(x+u) g(x+v) e^{\frac{-2 i}{\hbar} \omega(u, v)} \mathrm{d} u \mathrm{~d} v .
$$

In the following, we will use $\left(\mathcal{O}_{C}\left(\mathbb{R}^{2 n}\right), *_{\hbar}\right)$, a complete nuclear (LP)-algebra, as our model for a noncommutative euclidean space.

\subsection{Construction of a Q-algebra}

In this subsection, we will construct a Q-algebra, actually a differential graded algebra. (We say that a constant symplectic (Poisson) torus is a torus with a constant symplectic (Poisson) structure.) For convenience, we work explicitly on a 2-dimensional torus; the same method can be generalized to an $n$-dimensional torus without extra effort.

Let $\left(\mathbb{T}^{2}, \omega\right)$ be a 2-dimensional symplectic torus and $C^{\infty}\left(\mathbb{T}^{2}\right)$ be the algebra of smooth functions on $\mathbb{T}^{2}$. Denote $\mathcal{W}$ to be a trivial infinite-dimensional vector bundle over $\mathbb{T}^{2}$ with fiber isomorphic to $\mathcal{O}_{C}\left(\mathbb{R}^{2}\right)$. We consider the following algebra

$$
\Omega\left(\mathbb{T}^{2}, \mathcal{W}\right) \stackrel{\text { def }}{=} \Gamma^{\infty}\left(\wedge^{*} \mathbb{T}^{2}\right) \otimes \mathcal{O}_{C}\left(\mathbb{R}^{2}\right)
$$

where $\otimes$ is the projective tensor product with respect to the topologies. We remark that we need to replace the standard symplectic form in (3) by the symplectic form on

the torus, in order to include the information of the symplectic (Poisson) torus in the definition of $*_{\hbar}$ on $\mathcal{O}_{C}$.

The product on $\mathcal{A}$ is defined as

$$
f *_{\hbar} g(x, y) \stackrel{\text { def }}{=}\left(f(x) *_{\hbar} g(x)\right)(y)
$$

where $x$ and $y$ are coordinates on $\mathbb{T}^{2}$ and $\mathbb{R}^{2}, f(x)$ and $g(x)$ are restrictions of $f$ and $g$ to $x$, and the $*$ between $f(x)$ and $g(x)$ is the 'twisted product' defined in Theorem 2.1. $\Omega\left(\mathbb{T}^{2}, \mathcal{W}\right)$ can be viewed as the space of smooth sections of $\mathcal{W}$, and is naturally graded by the degree of differential forms. In the following, we will define a $Q$ operator on $\Omega\left(\mathbb{T}^{2}, \mathcal{W}\right)$.

We define

$$
Q \stackrel{d e f}{=} d x^{i} \wedge\left(\frac{\partial}{\partial x^{i}}-\frac{\partial}{\partial y^{i}}\right)
$$

where $x^{i}$ s are the coordinates on $\mathbb{T}^{2}$ and $y^{i}$ s are the coordinates on $\mathbb{R}^{2}$.

It is straightforward to check that $\operatorname{deg}(Q)=1$ and $Q^{2}=0$. Therefore, $\left(\Omega\left(\mathbb{T}^{2}, \mathcal{W}\right), Q\right)$ is a Q-algebra with $\omega=-\frac{i}{\hbar} d x^{1} \wedge d x^{2}$. Since $\omega$ is a center element in $\mathcal{A}$ and therefore we can view $\left(\Omega\left(\mathbb{T}^{2}, \mathcal{W}\right), Q\right)$ as a differential graded algebra. 


\subsection{Quantization}

In this part, we prove that the Q-algebra $\Omega\left(\mathbb{T}^{2}, \mathcal{W}\right)$ defined in the previous subsection is quasi-isomorphic to $C^{\infty}\left(\mathbb{T}^{2}\right)$ as differential graded algebras. By identifying the 0-th cohomologies of $(\mathcal{A}, Q)$ and $C^{\infty}\left(\mathbb{T}^{2}\right)$, we will obtain a quantization map.

Theorem 2.2 The differential algebra $\left(\Omega\left(\mathbb{T}^{2}, \mathcal{W}\right), Q\right)$ is acyclic and has 0-th cohomology equal to $C^{\infty}\left(\mathbb{T}^{2}\right)$.

Proof. The crucial part of the proof already appears in [5] for different purposes. By a change of coordinates $\tilde{x}=x-y, \tilde{y}=x+y$, we have the following observations.

1. $\Omega\left(\mathbb{T}^{2}, \mathcal{W}\right)=\Gamma^{\infty}\left(\wedge^{*} \mathbb{T}^{2}\right) \otimes \mathcal{O}_{C}\left(\mathbb{R}^{2}\right)$ is isomorphic to $\Gamma^{\infty}\left(\wedge_{-}^{*}\left(\mathbb{T}^{2} \times \mathbb{R}^{2}\right)\right) \otimes \mathcal{O}_{C}\left(\mathbb{R}^{2}\right)$, where $\wedge_{-}^{*}\left(\mathbb{T}^{2} \times \mathbb{R}^{2}\right)$ denotes the differential forms on $\mathbb{T}^{2} \times \mathbb{R}^{2}$ along the direction of $\tilde{y}$.

2. Under the above isomorphism, $Q$ is mapped to $\tilde{Q}=d \tilde{y} \wedge \frac{\partial}{\partial \tilde{y}}$.

The following are reasons for the acyclicity of $\tilde{Q}$.

1. Poincaré lemma for $\tilde{Q}$. It is easy to see that $\tilde{Q}$ is the de Rham differential along the $\tilde{y}$ direction on $\mathbb{T}^{2} \times \mathbb{R}^{2}$, and subspace of $\mathbb{T}^{2} \times \mathbb{R}^{2}$ along the $\tilde{y}$ direction is isomorphic to $\mathbb{R}^{2}$. Therefore, the Poincaré lemma is true on $\tilde{Q}$.

2. The homotopy formula constructed in the Poincaré lemma, i.e. $\psi(x)=\int_{0}^{1} \sum_{i} \phi_{i}(t x) x_{i} d t$ for $\phi=\sum_{i} \phi_{i}$ and $d \phi=0$. We know that $\phi_{i} \in \mathcal{O}_{C}\left(\mathbb{R}^{2}\right)$ implies $\psi \in \mathcal{O}_{C}\left(\mathbb{R}^{2}\right)$ by a simple estimation. (This has been observed in [5].)

Therefore, by the isomorphism between $Q$ and $\tilde{Q}$, we conclude that $Q$ is also acyclic. The 0 -th cohomology of $Q$ is equal to the solution of the following equation in $\Omega\left(\mathbb{T}^{2}, \mathcal{W}\right)$ :

$$
\left(\frac{\partial}{\partial x^{i}}-\frac{\partial}{\partial y^{i}}\right) f=0 . \quad i=1,2 .
$$

Obviously, the solutions of (4I) are functions on $\mathbb{T}^{2} \times \mathbb{R}^{2}$ of the form $g(x+y)$. Therefore, the map $\sigma: H^{0}\left(\Omega\left(\mathbb{T}^{2}, \mathcal{W}\right), Q\right) \rightarrow C^{\infty}(\mathbb{T}), \sigma(g)=\left.g\right|_{y=0}$, defines an isomorphism between the 0 -th cohomologies of $Q$ and $C^{\infty}\left(\mathbb{T}^{2}\right)$.

By Theorem 2.2, we define a quantization map $\mathcal{Q}=\sigma^{-1}: C^{\infty}\left(\mathbb{T}^{2}\right) \rightarrow H^{0}\left(\Omega\left(\mathbb{T}^{2}, \mathcal{W}\right), Q\right)$ by $\mathcal{Q}(f)(x, y) \stackrel{\text { def }}{=} f(x+y)$. A star product on $C^{\infty}\left(\mathbb{T}^{2}\right)$ can be defined as

$$
f \star_{\hbar} g(x) \stackrel{\text { def }}{=} \sigma\left(\mathcal{Q}(f) *_{\hbar} \mathcal{Q}(g)\right)
$$

By construction, $\star_{\hbar}$ is smooth with respect to $\hbar$.

According to Theorem 2.2 and the above construction of $\star_{\hbar}$, we have the following proposition. 
Proposition 2.3 If we view the algebra $\left(C^{\infty}\left(\mathbb{T}^{2}\right)\right.$, $\left.\star_{\hbar}\right)$ as a differential graded algebra with 0 differential and only nontrivial 0-degree component, the map $\mathcal{Q}:\left(C^{\infty}\left(\mathbb{T}^{2}\right), \star_{\hbar}, 0\right) \rightarrow$ $\left(\Omega\left(\mathbb{T}^{2}, \mathcal{W}\right), *_{\hbar}, Q\right)$ is a quasi-isomorphism.

We can look at the bundle $V$ on $\mathbb{T}^{2}, V=\mathcal{S}(\mathbb{R}) \times \mathbb{T}^{2} \rightarrow \mathbb{T}^{2}$. By Theorem 2.1, there is a natural representation of $\mathcal{O}_{C}$ on $\mathcal{S}(\mathbb{R})$. Hence, we obtain an action of $\Omega\left(\mathbb{T}^{2}, \mathcal{W}\right)$ on $V$ acting by pointwise multiplication. Accordingly, we have a representation of $\left(C^{\infty}\left(\mathbb{T}^{2}\right), \star_{\hbar}\right)$ by composing $R$ with $\mathcal{Q}$. By this representation, we can define an involution ${ }^{*}$ and a pre $C^{*}$-norm on $\left(C^{\infty}\left(\mathbb{T}^{2}\right), \star_{\hbar}\right)$ using Hilbert module techniques. It is easy to check that $\left(C^{\infty}(\mathbb{T}), \star_{\hbar},{ }^{{ }^{*} \hbar},\|\|_{\hbar}\right)$ satisfies the definition of a strict deformation quantization as defined in Rieffel [10].

Therefore, by using $Q$-algebras, we have obtained a strict deformation quantization of a Poisson torus.

Remark 2.4 Our results of strict deformation quantization of a Poisson torus actually coincides with Rieffel's [10] construction. What we have done here can be considered as a geometric explanation of Rieffel's method. Similarly, we can use this Q-algebra method to obtain all the examples constructed in Rieffel [10].

\section{$3 \quad$ Fock module and dg-modules}

In this section, we construct some quantization dg-modules of the Q-algebra $(\Omega(X, \mathcal{W}), \nabla, R)$. We first introduce the notion of a Fock module over the Q-algebra on a compact Kähler manifold.

Let $X$ be a compact Kähler manifold with $\operatorname{dim}_{\mathbb{C}}(X)=n$. At any $x$ of $X$, the tangent space $T_{x} X$ is equipped with a riemannian metric. We define an idempotent element

$p_{\hbar}=2^{n} \exp \left(-\frac{|y|^{2}}{\hbar}\right)$ in $\left(\mathcal{O}_{C}\left(T_{x} X\right), *_{\hbar}\right)$, where $|\cdot|$ denotes the riemannian metric. It is easy to check that $p_{\hbar} *_{\hbar} p_{\hbar}=p_{\hbar}$, and according to [7], Equation (27), $p_{\hbar} *_{\hbar} \bar{y}^{i}=0$ for $1 \leq i \leq n$. Theorem 4, 7] proves that $p_{\hbar} *_{\hbar} \mathcal{O}_{C}$ is a subspace of $\mathcal{S}\left(\mathbb{R}^{2 n}\right)$.

Furthermore $p_{\hbar}$ is a well defined function over $T X$ and therefore defines a smooth section of $\mathcal{W}$. We define a right projective $\Omega(X, \mathcal{W})$ module $\mathcal{F}$ by $p_{\hbar} *_{\hbar} \Omega(X, \mathcal{W})$.

On a Kähler manifold, there is a natural choice of a symplectic connection $\nabla$, the Levi-Civita connection, i.e. $\nabla=d+\Gamma$. The operator $Q$ on $\Omega(X, \mathcal{W})$ is written as

$$
Q(a)=d a+\frac{1}{\hbar}[\Gamma, a], \text { for all } a \in \Omega(X, \mathcal{W}),
$$

where $\Gamma=\Gamma_{i j} \bar{y}^{i} y^{j}$ and $\Gamma_{i j}=\bar{\Gamma}_{j i}$.

One can define a connection $\nabla_{\mathcal{F}}$ on $\mathcal{F}$ using $Q$ as follows,

$$
\nabla_{\mathcal{F}}\left(p_{\hbar} *_{\hbar} a\right)=p_{\hbar} *_{\hbar} Q\left(p_{\hbar}\right) *_{\hbar} a+p_{\hbar} *_{\hbar} Q(a) .
$$

It is straight forward to check that $Q\left(p_{\hbar}\right)=0$, so

$$
\nabla_{\mathcal{F}}\left(p_{\hbar} *_{\hbar} a\right)=p_{\hbar} *_{\hbar} Q(a)=p_{\hbar} *_{\hbar} d(a)+p_{\hbar} *_{\hbar}[\Gamma, a] .
$$


Noticing that $p_{\hbar} *_{\hbar} \bar{y}^{i}=0$, we have that

$$
\nabla_{\mathcal{F}}\left(p_{\hbar} *_{\hbar} a\right)=p_{\hbar} *\left(d a-a *_{\hbar}\left(\frac{1}{\hbar} \Gamma_{i j} \bar{y}^{i} *_{\hbar} y^{j}\right)\right) .
$$

Let $\Omega$ be the curvature form of $\nabla$. And we compute the curvature of $\nabla_{\mathcal{F}}$ to be

$$
\nabla_{\mathcal{F}}^{2}\left(p_{\hbar} *_{\hbar} a\right)=p_{\hbar} *_{\hbar}\left(-\frac{1}{\hbar} R\right)
$$

where $R$ is equal to $R_{i j} \bar{y}^{i} * y^{j}$.

We recall that $Q^{2}(a)=\frac{1}{\hbar}[R, a]$, and conclude that

Proposition 3.1 The Fock module $\left(\mathcal{F}, \nabla_{\mathcal{F}}\right)$ has zero curvature.

In the following, we want to twist this Fock module by a line bundle to get a quantization dg-module over $(\Omega(X, \mathcal{W}), \nabla, R)$. We consider a rank one right projective module $H_{m}$ of $C^{\infty}(X)$ with the curvature equal to $-\mathrm{im} \omega$. We identify $H_{m}$ with the space of $p_{m} C^{\infty}(X)$, where $p_{m}$ is a projective matrix valued in $C^{\infty}(X)$. Define $P_{m} \stackrel{\text { def }}{=} p_{\hbar} p_{m}$ in $\Omega(X, \mathcal{W})$. We notice that $p_{\hbar} *_{\hbar} p_{m}=p_{m} *_{\hbar} p_{\hbar}=p_{\hbar} p_{m}$ and $P_{m} *_{\hbar} P_{m}=P_{m}$. Therefore $\mathcal{F}_{m} \stackrel{\text { def }}{=} P_{m} *_{\hbar} \Omega(X, \mathcal{W})$ defines a right projective module over $\Omega(X, \mathcal{W})$ with the connection $\nabla_{m}$ equal to

$$
\nabla_{m}\left(P_{m} *_{\hbar} a\right)=P_{m} *_{\hbar} Q(a)+P_{m} *_{\hbar} \nabla_{H_{m}}\left(p_{m}\right) *_{\hbar} a .
$$

By a calculation similar to that used in the case of the Fock module, the curvature of $\mathcal{F}_{m}$ is equal to -im $\omega$. By Definition 1.3, $\mathcal{F}_{m}$ is a quantization dg-module over $(\Omega(X, \mathcal{W}), \nabla, R)$ when $\hbar=\frac{1}{m}$.

Proposition 3.2 The twisted Fock module $\mathcal{F}_{m}$ is a quantization dg-module over $(\Omega(X, \mathcal{W})$, $\nabla, R)$ when $\hbar=\frac{1}{m}$.

We recall the procedure for the Berezin-Toeplitz quantization of $\mathbb{C P}^{1}$. One considers the hyperplane bundle $H$, and its powers $H^{\otimes l}$ over $\mathbb{C P}^{1}$. According to the Riemann-Roch theorem, for $l \geq 0$, the space $B_{l}$ of holomorphic sections of $H^{\otimes l}$ is finite. One considers the action of $C^{\infty}\left(\mathbb{C P}^{1}\right)$ on $B_{l}$ by the composition of the multiplication with projection. When $l \rightarrow \infty$, this defines a deformation quantization of $\mathbb{C P}^{1}$ (see [2]). The action of $C^{\infty}\left(\mathbb{C P}^{1}\right)$ on $B_{l}$ defines a representation of a fuzzy sphere. In the following we study the twisted Fock module $\mathcal{F}_{m}$ introduced in Proposition 3.2 in the case of $\mathbb{C P}^{1}$. We relate the twisted Fock module $\mathcal{F}_{m}$ to the Berezin-Toeplitz quantization of $\mathbb{C P}^{1}$.

We start by looking at the Fock module $\mathcal{F}$. The module $\mathcal{F}$ is the space of sections of an infinite dimensional vector bundle over the manifold $X$ with fiber isomorphic to $p_{\hbar} *_{\hbar} \mathcal{O}_{C}$. This bundle is called the Fock bundle of $X$. In the next two paragraphs, we construct a dense subbundle of the Fock bundle. 
At each point $x \in X$, we look at the subspace $\mathcal{T}_{x}^{k}$ of the fiber $\mathcal{F}_{x}$ of the Fock bundle, which is the span of $p_{\hbar} *_{\hbar} y_{i_{1}} *_{\hbar} \cdots *_{\hbar} y_{i_{k}}$, where $1 \leq i_{1}, \cdots, i_{k} \leq n$. $\mathcal{T}_{x}^{k}$ is a finite dimensional subspace of $\mathcal{F}_{x}$. As $p_{\hbar} *_{\hbar} \bar{y}_{i}=0$, the direct sum of $\mathcal{T}_{x}^{k}$ is equal to the span of $p_{\hbar} *_{\hbar} f$ over all polynomials $f(y, \bar{y})$. Since $p_{\hbar} *_{\hbar} \mathcal{O}_{C}$ is a subspace of $\mathcal{S}$, the linear span of $p_{\hbar} *_{\hbar} f(y, \bar{y})$ over all polynomials $f(y, \bar{y})$ is dense in $p_{\hbar} *_{\hbar} \mathcal{O}_{C}$. Therefore, $\oplus_{k} \mathcal{T}_{x}^{k}$ is dense in $\mathcal{F}_{x}$.

Fixing $k$, we look at the collection of $\mathcal{T}_{x}^{k}$ over $X$. This is a finite dimensional vector bundle over $X$. The space of sections of this bundle is denoted by $\mathcal{T}^{k}$. According to the above fiberwise discussion, on $\mathcal{T}_{x}^{k}$, we know that $\Omega\left(X, \oplus_{k} \mathcal{T}^{k}\right)$ is dense in $\mathcal{F}$. We notice that $\nabla_{\mathcal{F}}$ restricts to a connection on $\mathcal{T}^{k}$ as a right $C^{\infty}(X)$ module with the curvature equal to $-R / \hbar$. The Chern character of $\mathcal{T}^{k}$ is equal to $C h\left(\mathcal{T}^{k}\right)=\operatorname{tr}(\exp (-k R / \hbar))$. Using the fact that $p_{\hbar} *_{\hbar} y_{i}^{* k} *_{\hbar}\left(\bar{y}_{i} *_{\hbar} y_{i}\right)=k \hbar\left(p_{\hbar} *_{\hbar} y_{i}^{* k}\right)$, we compute the Chern character of $\mathcal{T}^{k}$ to be equal to $C h(-T X)^{k}$, where $C h(-T X) \in H^{2 \bullet}(X)$ is the Chern character of $-T X$.

In the following we look at a special case, $\mathbb{C P}^{1}$. Since $\mathbb{C P}^{1}$ is one dimensional, $\mathcal{T}^{k}$ is the space of sections of a rank one bundle over $\mathbb{C P}^{1}$. According to the above calculation of Chern character, we know that $\mathcal{T}^{k}$ corresponds to the bundle $K^{\otimes k}$, where $K$ is the cotangent bundle of $\mathbb{C P}^{1}$. We conclude that in the case of $\mathbb{C P}^{1}$, the Fock module $\mathcal{F}$ contains a dense subspace $\mathcal{T}$ which consists of sections of

$$
\bigoplus_{k \geq 0} \Omega^{*}\left(\mathbb{C P}^{1}\right) \otimes K^{\otimes k}
$$

As a vector bundle over $\mathbb{C P}^{1}$, the module $\mathcal{F}_{m}$ can be viewed as the tensor of the Fock bundle $\mathcal{F}$ with the line bundle $H_{m}$. According to the above analysis, we know that $\mathcal{F}_{m}$ contains a dense subspace $F_{m}$ which consists of sections of

$$
\left(\bigoplus_{k \geq 0} \Omega^{*}\left(\mathbb{C P}^{1}\right) \otimes K^{\otimes k}\right) \otimes H_{m}
$$

In the case of $\mathbb{C P}^{1}$, the line bundle $H_{m}$ is the $\mathrm{m}$-th power of the dual bundle of the hyperplane bundle $H$ over $\mathbb{C P}^{1}$ because its curvature is equal to $-\mathrm{im} \omega$. The canonical line bundle $K$ is dual to the square of the hyperplane bundle $H$ because its curvature is equal to $-2 i \omega$. Therefore, the above dense subspace $F_{m}$ of the twisted Fock module $\mathcal{F}_{m}$ can be identified with

$$
\bigoplus_{k \geq 0} \Omega^{*}\left(\mathbb{C P}^{1}\right) \otimes H^{\otimes(-m-2 k)}
$$

We conclude that the dual bundle of the twisted Fock module $\mathcal{F}_{m}$ contains the direct sum of bundles $H^{\otimes m} \oplus H^{\otimes m+2} \oplus \cdots$.

Proposition 3.3 The fuzzy sphere acts on the dual of the twisted Fock module $\mathcal{F}_{m}$ with the representation $\oplus_{k \geq 0} B_{m+2 k}$

In the above, we introduced and studied the twisted Fock module over the Q-algebra $(\Omega(X, \mathcal{W}), \nabla, R)$, which is a special example of quantization $d g$-modules. The twisted 
Fock module is an infinitely generated projective module over $\Omega(X, \mathcal{W})$. One must ask whether there are any finitely generated projective quantization dg-modules.

The answer is affirmative in the case of a 2-dimensional torus $\mathbb{T}^{2}$ with a constant Poisson structure. According to Proposition 2.3, the Q-algebra $\Omega\left(\mathbb{T}^{2}, \mathcal{W}\right)$ is quasi-isomorphic to the algebra of the corresponding quantum torus by the map $\mathcal{Q}: C^{\infty}\left(\mathbb{T}^{2}\right) \rightarrow \Omega\left(\mathbb{T}^{2}, \mathcal{W}\right)$. Given a projection $P$ of the quantum torus, we define a right projective module $M_{P}$ of $\Omega\left(\mathbb{T}^{2}, \mathcal{W}\right)$ by $\mathcal{Q}(P) *_{\hbar} \Omega\left(\mathbb{T}^{2}, \mathcal{W}\right)$. It is straightforward to check that $M_{P}$ defines a finitely generated projective quantization dg-module by the equation $Q(\mathcal{Q}(P))=0$. Unfortunately, we do not know whether a general symplectic manifold has any finitely generated projective dg-modules. We end this note with the following conjecture:

Conjecture 3.4 For a symplectic manifold $M$, the Q-algebra $(\Omega(M, \mathcal{W}), \nabla, R)$ has finitely generated projective quantization dg-modules. Furthermore, the K-group of finitely generated projective quantization dg-modules over $(\Omega(M, \mathcal{W}), \nabla, R)$ is isomorphic to the $K$ group of finitely generated projective modules over $C^{\infty}(M)$.

\section{References}

[1] Alexandrov, M., Kontsevich, M., Schwarz, A., and Zaboronsky, O., The geometry of the master equation and topological quantum field theory, Int. J. Mod. Phys., A12(1997), 1405-1403.

[2] Bordemann, M., Meinrenken, E., and Schlichenmaier, M., Toeplitz quantization of Kähler manifolds and $\mathrm{gl}(N), N \rightarrow \infty$ limits, Comm. Math. Phys. 165 (1994), no. 2, $281-296$.

[3] Cattaneo, A., and Felder, G., Poisson sigma models and deformation quantization, Mod. Phys. Lett., A16(2001), 179-190.

[4] Connes, A. Noncommutative geometry. Academic Press, Inc., San Diego, CA, 1994.

[5] Dubois-Violette, M., Kriegl, A., Maeda, Y., and Michor, P., Smooth *-algebras. Noncommutative geometry and string theory (Yokohama, 2001). Progr. Theoret. Phys. Suppl. No. 144 (2001), 54-78.

[6] Fedosov, B., Deformation Quantization and Index Theory, Mathematical Topics, 9, Akademie Verlag, Berlin, 1996.

[7] Gracia-Bondia, J., Várilly, J., Algebras of distributions suitable for phase-space quantum mechanics. I., J. Math. Phys. 29 (1988), no. 4, 869-879.

[8] Karabegov, A., On Fedosov's approach to deformation quantization with separation of variables, Conférence Moshé Flato 1999, Vol. II (Dijon), 167-176, Math. Phys. Stud., 22, Kluwer Acad. Publ., Dordrecht, 2000. 
[9] Kontsevich, M., Deformation quantization of Poisson manifolds, Lett. Math. Phys. 66 (2003), no. 3, 157-216.

[10] Rieffel, M., Deformation quantization for actions of $\mathbb{R}^{d}$, Mem. Amer. Math. Soc. 106 (1993), no. 506.

[11] Schwarz, A., Geometry of Batalin-Vilkovisky quantization, Comm. Math. Phys., 155(1993), 249-260.

[12] Schwarz, A., Semiclassical approximation in Batalin-Vilkovisky Formalism, Comm. Math. Phys., 158(1993), 375-396.

[13] Schwarz, A., Noncommutative supergeometry and duality, Lett. Math. Phys. 50 (1999), no. 4, 309-321.

[14] Schwarz, A., Noncommutative supergeometry, duality and deformations, Nuclear Phys. B 650 (2003), no. 3, 475-496.

Department of Mathematics, University of California at Davis, Davis, CA, 95616, U.S.A. xtang@math.ucdavis.edu 